Original Article

\title{
Lower-limb muscle strength according to bodyweight and muscle mass among middle age patients with type 2 diabetes without diabetic neuropathy
}

\author{
Fuminari Asada ${ }^{1)}$, Takuo Nomura ${ }^{2}{ }^{*}$, Mitsuo Tagami ${ }^{3,4)}$, Masashi Kubota ${ }^{1)}$, \\ Makoto Ohashi ${ }^{1)}$, Makoto Nomura ${ }^{5)}$ \\ 1) Research Center for the Health Promotion and Employment Support, Osaka Rosai Hospital, Japan \\ 2) Department of Rehabilitation Sciences, Faculty of Allied Health Sciences, Kansai University of \\ Welfare Sciences: 1-11, 3-chome, Asahigaoka, Kashiwara-city, Osaka 582-0026, Japan \\ 3) Central Department of Rehabilitation, Osaka Rosai Hospital, Japan \\ 4) Present address: Central Department of Rehabilitation, Chugoku Rosai Hospital, Japan \\ 5) Center for Diabetes Mellitus, Osaka Rosai Hospital, Japan
}

\begin{abstract}
Purpose] This study assessed the effects of type 2 diabetes without diabetic polyneuropathy on muscle strength according to body composition in middle age patients. [Subjects and Methods] This study included 45 nondiabetic individuals (control group) and 50 patients with type 2 diabetes (DM group), 40 to 64 years of age. The body composition was examined, including the leg muscle volume (LMV), which was the sum of the lower-limb muscle mass. The muscle strength was also examined, and the knee extension force (KEF), ankle dorsiflexion force (ADF). The KEF and ADF were normalized to the bodyweight, and the total leg muscle force (TLMF) were calculated by combining the KEF and ADF. The leg muscle quality (LMQ) was calculated as the TLMF normalized with the LMV. [Results] While no significant differences were found in the LMV between groups, the body mass index were higher in the DM group than in the control group. Significant differences were observed in \%KEF, \%ADF, and the LMQ in the DM group, with $15.8 \%, 18.7 \%$, and $11.5 \%$ lower values than those in the control group, respectively. [Conclusion] The results of this study may demonstrate that muscle weakness occurs before diabetes progresses to a severe condition.

Key words: Diabetes mellitus, Muscle weakness, Middle aged
\end{abstract}

(This article was submitted Mar. 3, 2017, and was accepted Apr. 17, 2017)

\section{INTRODUCTION}

Falling birth rates and increasingly aging populations have resulted in aging labor forces in many developed countries ${ }^{1)}$. Gent et al. ${ }^{2}$ studied the anaerobic capacity $\left(\mathrm{kJ} \mathrm{kg}^{-1}\right)$ in 156 men and 17 women 35 to 65 years of age and reported an 8.0 $\pm 3.3 \%$ decrease in capacity with each 10 year age increment. Yamada et al. ${ }^{3)}$ reported that the limb skeletal muscle mass decreased 10.8 and $6.4 \%$ in men and women, respectively, above 35 years of age (40-44 to 75-79 years). While the pattern of decrease varied, an approximately $20 \%$ decrease in the physical work capacity due to decreased anaerobic capacity and skeletal muscle mass was associated with an increased incidence of work-related injuries and illness in persons 40 to 60 years of age ${ }^{4}$. Goetzel et al. ${ }^{5)}$ observed that the hospital visiting rates and annual medical costs were highest among over bodyweight individuals (body mass index [BMI] $25.0-29.9 \mathrm{~kg} / \mathrm{m}^{2}$ ), followed by obese individuals (BMI $\geq 30.0 \mathrm{~kg} / \mathrm{m}^{2}$ ), compared

*Corresponding author. Takuo Nomura (E-mail: tnomura@tamateyama.ac.jp; nomurata1017@yahoo.co.jp)

(C2017 The Society of Physical Therapy Science. Published by IPEC Inc.

This is an open-access article distributed under the terms of the Creative Commons Attribution Non-Commercial No Derivatives (by-nc-nd) License. (CC-BY-NC-ND 4.0: https://creativecommons.org/licenses/by-nc-nd/4.0/) 
with those of normal bodyweight (BMI $18.0-25.0 \mathrm{~kg} / \mathrm{m}^{2}$ ) in 10,026 workers from multiple occupations and work places, and that the presenteeism rates of workers increased with the BMI. Therefore, maintaining worker health and maintaining or improving the physical work capacity are essential to prevent decreased anaerobic capacity and skeletal muscle mass.

The number of patients with diabetes is increasing worldwide. Currently, one in 11 adults develops diabetes, a rate expected to increase to one in 10 adults by $2040^{6}$. In recent years, patients with diabetes have been reported to develop motor dysfunctions ${ }^{7)}$. Andersen et al. ${ }^{8)}$ evaluated 36 patients with type 2 diabetes (mean age: 59 years) and 36 unaffected individuals (mean age: 58 years), and reported increased lower-limb muscle weakness in the diabetes group. A comparison of the magnitude of lower-limb muscle weakness between the diabetes and control groups revealed significantly decreased ankle flexor (17\%), ankle extensor (14\%), and knee flexor (14\%) forces, and a decreasing tendency in the knee extensor force (7\%) in the diabetes group. IJzerman et al. ${ }^{9}$ ) examined lower-limb muscle strength in 98 patients with diabetic polyneuropathy (DPN) (mean age: 67 years), 39 type 2 diabetic patients without DPN (mean age: 62 years), and 19 healthy controls (mean age: 68 years) 50 years of age and older. After normalizing for bodyweight, ankle plantar flexion, ankle dorsiflexion, knee extensor, and knee flexor forces were at least $30 \%$ in patients with type 2 diabetes without DPN compared to non-diabetic controls.

Previous studies have examined the effects of diabetes on lower-limb muscle strength in patients with type 2 diabetes, including individuals complicated by DPN and 65 years of age and older; however, no study has examined in detail the effects of diabetes on lower-limb muscle strength in only middle-aged patients with type 2 diabetes not complicated by DPN. In addition, complications typically develop with progression to severe diabetes; thus, the effects of diabetes on lower-limb muscle strength before this progression remain unknown. It is therefore important to clarify the effects of diabetes on muscle strength among middle-aged workers in order to better assess the physical work capacity of the working generation.

This study examined the body composition and lower-limb muscle strength in middle-aged patients with type 2 diabetes without DPN, as well as in non-diabetic control subjects, in order to clarify the effects of type 2 diabetes on muscle strength according to differences in the muscle mass.

\section{SUBJECTS AND METHODS}

This study included subjects without diabetes (control group) and with type 2 diabetic (DM group) between 40 and 64 years of age, without any limitations on performing daily and physical activities. Subjects in the control group were non-diabetic individuals working for a company that had requested that we provide exercise and nutrition education or those who visited the outpatient clinic of the hospital for screening for lifestyle-related diseases. Subjects were included in the DM group if they were diagnosed with type 2 diabetes, were hospitalized or visiting the hospital for diabetes, and were prescribed active exercise therapy by their physicians. Individuals were excluded if they had end-stage renal disease requiring dialysis, were already on dialysis, were receiving treatment for proliferative retinopathy, had a history of proliferative retinopathy, had serious complications such as DPN, or were in the DM group and had a history of complications. In both groups, individuals who engaged in occupations associated with heavy exertion (e.g., farming, forestry, and fishery jobs, which require significant muscle use) were excluded. In addition, individuals were excluded if they had any disease affecting their locomotion or physical function (e.g., cervical vascular disorder, spinal cord injury, or osteoarthrosis) or a history of those diseases.

Exercise habits, body composition, and lower-limb muscle strength were measured in both groups. Exercise was defined as more than 30 minutes of physical activity at a time, more than twice a week, as described by the National Health and Nutrition Survey in Japan ${ }^{10)}$. A bioelectrical impedance analysis was used to estimate the body composition. All facilities used a body composition analyzer (InBody720, Biospace Corp., Seoul, South Korea) for measurements after moistening the subject's soles and palms with a moist towelette to minimize measurement errors due to dry skin ${ }^{11)}$. The skeletal muscle volume (SMV) of the whole body, the amount of body fat (ABF) of the whole body, and the leg muscle volume (LMV), which was the sum of the lower-limb muscle mass without any body fat, were measured. The knee extension force (KEF) and ankle dorsiflexion force (ADF) were measured to define lower-limb muscle strength. The non-dominant leg (the pivot leg, i.e., the leg one would not kick a ball with) was designated as the leg from which the measurements are to be performed. The isometric KEF with hip and knee flexion angles of $90^{\circ}$ in the sitting position and isometric ADF with ankle joint angles of $0^{\circ}$ in the supine position were measured using a hand-held dynamometer ( $\mu$ Tas F-01, Anima Corp., Tokyo, Japan). A fixed belt was used to enhance the repeatability of these measurements ${ }^{12}$. The muscle strength values were normalized by dividing by the subject's bodyweight in order to examine percentages. The assessed values were the total leg muscle force (TLMF), defined as the sum of the KEF and ADF, and the leg muscle quality (LMQ), defined as dividing the TLMF by the LMV. In the DM group, data including the estimated duration of diabetes and an index of diabetic control were collected. Patients were considered to be positive for DPN if they met two or more of three following criteria: subjective symptoms possibly due to DPN (e.g., numbness, pain, or dysesthesia in the toe or the sole), decreased sense of vibration at the medial malleolus of both legs, or loss or lessening of the Achilles tendon reflex of both legs ${ }^{13)}$.

Data were collected from April 2010 to December 2012. Physical therapists at Osaka Rosai Hospital (Osaka prefecture) and Tohoku Rosai Hospital (Miyagi prefecture) collected data from subjects in the control group. Physical therapists at Osaka Rosai Hospital (Osaka prefecture), Kansai Rosai Hospital (Hyogo prefecture), Tokyo Rosai Hospital (Tokyo prefecture), Tohoku Rosai Hospital (Miyagi prefecture) and Hokkaido Chuo Rosai Hospital (Hokkaido prefecture) collected data from 
Table 1. Comparison of the control group and the DM group

\begin{tabular}{|c|c|c|}
\hline \multirow{2}{*}{ Parameter (unit) } & Control group $(n=45)$ & DM group $(\mathrm{n}=50)$ \\
\hline & Mean SD & Mean SD \\
\hline \multicolumn{3}{|l|}{ Characteristics } \\
\hline Female/Male (n) & $15 / 30$ & $12 / 38$ \\
\hline Age (years) & $53.2 \pm 6.3$ & $53.9 \pm 6.6$ \\
\hline Body height $(\mathrm{cm})$ & $164.9 \pm 9.6$ & $164.6 \pm 6.8$ \\
\hline Bodyweight (kg) & $65.2 \pm 11.7$ & $72.6 \pm 10.8^{*}$ \\
\hline Body mass index $\left(\mathrm{kg} / \mathrm{m}^{2}\right)$ & $23.8 \pm 3.5$ & $26.8 \pm 4.2^{* *}$ \\
\hline Systolic blood pressure ( $\mathrm{mmHg}$ ) & $135.2 \pm 19.6$ & $133.1 \pm 16.6$ \\
\hline Diastolic blood pressure (mmHg) & $82.1 \pm 12.7$ & $82 \pm 10.3$ \\
\hline Heart rate $(\mathrm{b} / \mathrm{min})$ & $73.3 \pm 18.7$ & $76.2 \pm 13.9$ \\
\hline Presence of exercise habits (n) & 12 & 13 \\
\hline \multicolumn{3}{|c|}{ Body consumption and lower extremity muscle strength } \\
\hline Skeletal muscle volume (kg) & $26 \pm 5.2$ & $27.9 \pm 4.4$ \\
\hline Amount of body fat $(\mathrm{kg})$ & $17.9 \pm 7.2$ & $22.3 \pm 8.8^{*}$ \\
\hline Leg muscle volume ( $\mathrm{kg})$ & $7.5 \pm 1.6$ & $7.7 \pm 1.2$ \\
\hline Knee extension force (kgf) & $36.9 \pm 12.4$ & $34.6 \pm 9.4$ \\
\hline$\%$ Knee extension force $(\%)$ & $57.3 \pm 18.2$ & $48.2 \pm 13.4^{*}$ \\
\hline Ankle dorsiflexion force (kgf) & $21.2 \pm 3.9$ & $19.1 \pm 6.2$ \\
\hline$\%$ Ankle dorsiflexion force $(\%)$ & $33 \pm 6.1$ & $26.8 \pm 8.5^{* *}$ \\
\hline Total leg muscle force (kgf) & $58.1 \pm 14.7$ & $53.7 \pm 13.7$ \\
\hline Leg muscle quality & $7.8 \pm 1.6$ & $6.9 \pm 1.6^{*}$ \\
\hline
\end{tabular}

SD: standard deviation; ${ }^{*} \mathrm{p}<0.05 ;{ }^{* *} \mathrm{p}<0.001$; DM: diabetes mellitus; $\%$ Knee extension force: knee extension force $(\mathrm{KEF}) /$ bodyweight $\times 100$; \%Ankle dorsiflexion force: ankle dorsiflexion force (ADF)/ bodyweight $\times 100$; Total leg muscle force (TLMF): KEF+ADF; Leg muscle quality: TLMF/leg muscle volume

patients in the DM group. A measurement manual was prepared for data collection to ensure standard procedure and measurement methods. This study was approved by the Ethical Review Board of all five facilities to which the collaborating physical therapists belonged. Written consent was obtained from all subjects in this study after providing oral and written explanations.

Researchers not involved in the data collection performed the analyses. Continuous variables were compared between the groups using an unpaired t-test after confirming the normality of the data distribution using the Shapiro-Wilk test. Chi-square tests were used for comparison of nominal variables. The IBM SPSS Statistics for Windows version 19.0 software program (IBM Corporation, Armonk, NY, USA) was used for all analysis, with a $5 \%$ significance level.
Table 2. Clinical characteristics of study subjects with diabetes mellitus

\begin{tabular}{lc}
\hline \multicolumn{1}{c}{ Parameter (units) } & Mean SD \\
\hline Diabetes duration (years) & $8.3 \pm 7.9$ \\
Fasting plasma glucose (mg/dl) & $162 \pm 58.9$ \\
HbAlc (\%) & $8.8 \pm 2.3$ \\
Total cholesterol (mg/dl) & $203.1 \pm 34.6$ \\
Triglyceride (mg/dl) & $178.2 \pm 123.9$ \\
LDL-cholesterol (mg/dl) & $126.2 \pm 34.4$ \\
HDL- cholesterol (mg/dl) & $48.5 \pm 11.8$ \\
Serum creatinine (mg/dl) & $0.79 \pm 0.24$ \\
\hline
\end{tabular}

$\mathrm{n}=50 ; \mathrm{SD}$ : standard deviation

\section{RESULTS}

The characteristics of subjects in both groups and the results of the body composition and muscle strength assessments are shown in Table 1. The clinical characteristics of patients in the DM group are shown in Table 2. There were no significant differences in age, gender, blood pressure, heart rate, or exercise habits between the control and DM groups. However, the bodyweight and body mass index (BMI) were significantly higher in the DM group than in the control group ( $p<0.01$ and $p<0.001$, respectively). Additionally, there were no significant differences in the KEF, ADF, and TLMF between the groups. However, $\% \mathrm{KEF}$ and \%ADF were significantly lower in the DM group than in the control group $(\mathrm{p}<0.01$ and $\mathrm{p}<0.001$, respectively).

The amount of body fat in the DM group was significantly higher than that in the control group $(\mathrm{p}<0.05)$, while there were no significant differences in the SMV and LMV between both groups. The LMQ was significantly lower in the DM group than in the control group $(\mathrm{p}<0.01)$. The KEF, ADF, and TLMF in the DM group were $6.2 \%, 9.9 \%$, and $7.5 \%$ lower than those in the control group, respectively, with a tendency toward being lower. However, \%KEF, \%ADF, and the LMQ in the DM group were $15.8 \%, 18.7 \%$, and $11.5 \%$ lower than those in the control group, respectively, which were significantly different $(\mathrm{p}<0.01, \mathrm{p}<0.001$, and $\mathrm{p}<0.01$, respectively). 


\section{DISCUSSION}

This study compared the body composition and lower-limb muscle strength between DM and control groups consisting of middle-aged subjects not complicated by DPN in order to clarify the effects type 2 diabetes on muscle strength according to the muscle mass. Several factors may contribute to muscle strength weakness in patients with diabetes. A lack of exercise and the decline of physical activities may significantly decrease muscle strength. Moreover, an abnormal mitochondrial function, abnormal free fatty acid metabolism, and an inadequate increase in the microvascular blood supply during exercise are also likely to affect the muscle function ${ }^{14,15)}$.

The mean BMI in the DM group $\left(26.8 \pm 4.2 \mathrm{~kg} / \mathrm{m}^{2}\right)$ was significantly higher than that in the control group $(23.8 \pm 3.5 \mathrm{~kg} /$ $\mathrm{m}^{2}$ ). The mean BMI among Japanese adult individuals is $23 \mathrm{~kg} / \mathrm{m}^{2},{ }^{10)}$; thus, the physical constitution of subjects in the control group in this study were consistent with that of the general Japanese population. The initial data from the Japan Diabetes Complication Study (JDCS) ${ }^{16}$ ), which commenced in 1996, of approximately 2,200 Japanese patients with type 2 diabetes demonstrated that the mean BMI of diabetic patients (mean BMI of $23 \mathrm{~kg} / \mathrm{m}^{2}$ ) did not significantly differ from that of non-diabetic individuals. However, the Japan Diabetes Clinical Data Management study (JDDM) group reported ${ }^{17}$ ) an increased BMI in patients with type 2 diabetes enrolled between 2000 and 2009, with a mean BMI of $25.6 \pm 4.4 \mathrm{~kg} / \mathrm{m}^{2}$ in 438 patients enrolled from 2008 to 2009. In recent years, the number of obese Japanese patients with type 2 diabetes (defined as BMI $25 \mathrm{~kg} / \mathrm{m}^{2}$ or above) ${ }^{18)}$, has increased ${ }^{19)}$; thus, the physical constitution of subjects in the DM group in this study may reflect this recent trend. Comparisons of muscle strength were often performed using the patient's strength normalized to bodyweight, and this normalization muddles interpretation of the results when study populations involve groups with a high BMI. The muscle strength and bodyweight should therefore be used carefully in middle age patients with type 2 diabetes. Approximately $25 \%$ of Japanese individuals 40 to 69 years of age exercised regularly ${ }^{20)}$, and $26 \%$ of subjects in both the control and DM groups had regular exercise habits in this study. Therefore, the subjects in the current study had physical constitutions and exercise habits similar to those of the general Japanese population.

In the DM group, \% KEF and \% ADF were approximately 15-20\% lower than those in the control group, which were statistically significant. After normalizing for bodyweight, the magnitude of lower-limb muscle weakness in this study was similar to the data previously reported by Andersen et al. in subjects complicated by DPN. In this study, lower-limb muscle strength was measured using an isometric protocol, while Andersen et al. used an isokinetic protocol. IJzerman et al. ${ }^{9)}$ used the same isometric protocol as that used in our study to examine lower-limb muscle strength and reported a larger magnitude of muscle weakness than observed in our study. Measurement differences between the isokinetic and isometric protocols should be assessed in future studies. Regardless, the degree of lower-limb muscle weakness in the DM group observed in this study appears to be plausible. The mean bodyweight and BMI in the DM group was significantly higher than that of the control group; however, the subjects in this group would not be classified as having sarcopenic obesity ${ }^{21)}$ because the SMV did not significantly differ between both groups. Moreover, the significant difference in lower-limb muscle strength normalized by bodyweight observed between the groups was possibly due to the increased BMI in the DM group compared to that of the control group. However, the LMQ was significantly lower in the DM group than in the control group; thus, it appears likely that the abnormal muscular function ${ }^{14,15)}$ was due to the presence of DM.

Maintaining muscle strength is important for performing tasks as well as maintaining activities of daily living (ADL) ${ }^{22)}$. Hasegawa et al. ${ }^{23)}$ investigated muscle strength and independence in daily life in elderly people 81 to 89 years of age and reported that the lower limit of knee extension muscle strength necessary for independent ADL was approximately $30 \%$ $(2.8 \mathrm{~N} / \mathrm{kg})$. Park et al. ${ }^{24}$ ) observed a decreased muscle quality in patients with diabetes compared to control subjects in a study examining grip strength and the muscle quality of knee extension muscle strength (the ratio of muscle strength to muscle mass) in 485 patients with type 2 diabetes 70 to 79 years of age and 2,133 non-diabetic subjects. In a separate cohort study, Park et al. ${ }^{25}$ ) reported that the decrease in muscle strength, muscle mass, and muscle quality after three years was greater in patients with diabetes than in control subjects. In Japan, the growth of the elderly population has resulted in an increasing number of individuals requiring nursing care, reaching 5,643,155 in 2013, with this number forecasted to continue to increase $^{26)}$. This study included middle-aged patients with DM. Although the decrease in muscle strength was not sufficient to interrupt ADL, early intervention may be important for preventive care. IJzerman et al. ${ }^{27)}$ reported that patients with type 2 diabetes fatigue easily when required to use muscle strength during isokinetic exercises, compared to control subjects; thus, patients with diabetes should be closely monitored to prevent a decreased physical work capacity. In addition, muscle weakness may be a marker of an increased risk of insulin resistance ${ }^{28)}$; therefore, the measurement of muscle strength may be useful for controlling diabetes, as well as maintaining the physical work capacity.

There were some limitations associated with this study. While this study excluded individuals engaged in work expected to result in heavy exertion, we did not survey the participants' occupations details. In addition, we did not assess past exercise habits; thus, the effects of physical activities or exercise habits performed during adolescence, the 20s to 30 s, and before starting this study on the results of this study are unknown. The types of exercise habits (e.g., aerobic or resistance exercise) were also not investigated. Finally, the subjects' dietary habits were not examined in this study, although nutrition is also associated with decreased muscle mass and muscle weakness ${ }^{29}$. 


\section{ACKNOWLEDGEMENT}

This research was supported by research funds to promote the hospital functions of the Japan Labour Health and Welfare Organization. The authors thanks Kenichiro TAKANO from Kansai Rosai Hospital, Tomonori SATOH from Tohoku Rosai Hospital, Kayo KAWAMATA from Tokyo Rosai Hospital, Kazushi SAKAMOTO from Hokkaido Chuo Rosai Hospital, and Shinji HIRABAYASHI and Takuya KITAGUCHI from Osaka Rosai Hospital for their cooperation with this study.

\section{REFERENCES}

1) United Nations Department of Economics and Social Affairs: The 2015 Revision of World Population Prospects: http://esa.un.org/unpd/wpp/2015. http://esa. un.org/unpd/wpp/ (Accessed Nov. 8, 2016)

2) Gent DN, Norton K: Aging has greater impact on anaerobic versus aerobic power in trained masters athletes. J Sports Sci, 2013, 31: 97-103. [Medline] [CrossRef]

3) Yamada M, Moriguch Y, Mitani T, et al.: Age-dependent changes in skeletal muscle mass and visceral fat area in Japanese adults from 40 to 79 years-of-age. Geriatr Gerontol Int, 2014, 14: 8-14. [Medline] [CrossRef]

4) Kenny GP, Yardley JE, Martineau L, et al.: Physical work capacity in older adults: implications for the aging worker. Am J Ind Med, 2008, 51: 610-625. [Medline] [CrossRef]

5) Goetzel RZ, Gibson TB, Short ME, et al.: A multi-worksite analysis of the relationships among body mass index, medical utilization, and worker productivity. J Occup Environ Med, 2010, 52: S52-S58. [Medline] [CrossRef]

6) International Diabetes Federation: Diabetes Atlas, 7th edition 2015. https://www.idf.org/diabetesatlas; 2015 (Accessed Nov. 8, 2016)

7) Andersen H: Motor dysfunction in diabetes. Diabetes Metab Res Rev, 2012, 28: 89-92. [Medline] [CrossRef]

8) Andersen H, Nielsen S, Mogensen CE, et al.: Muscle strength in type 2 diabetes. Diabetes, 2004, 53: 1543-1548. [Medline] [CrossRef]

9) IJzerman TH, Schaper NC, Melai T, et al.: Lower extremity muscle strength is reduced in people with type 2 diabetes, with and without polyneuropathy, and is associated with impaired mobility and reduced quality of life. Diabetes Res Clin Pract, 2012, 95: 345-351. [Medline] [CrossRef]

10) Ministry of Health Labour and Welfare: National Health and Nutrition Survey. http://www.mhlw.go.jp/seisakunitsuite/bunya/kenkou_iryou/kenkou/kenkounippon21/en/eiyouchousa/index.html (Accessed Nov. 8, 2016)

11) Ogawa H, Fujitani K, Tsujinaka T, et al.: InBody 720 as a new method of evaluating visceral obesity. Hepatogastroenterology, 2011, 58: 42-44. [Medline]

12) Katoh M, Isozaki K, Sakanoue N, et al.: Reliability of isometric knee extension muscle strength measurement using a hand-held dynamometer with a belt: a study of test-retest reliability in healthy elderly subjects. J Phys Ther Sci, 2010, 22: 359-363. [CrossRef]

13) Yasuda H, Sanada M, Kitada K, et al.: Rationale and usefulness of newly devised abbreviated diagnostic criteria and staging for diabetic polyneuropathy. Diabetes Res Clin Pract, 2007, 77: S178-S183. [Medline] [CrossRef]

14) Womack L, Peters D, Barrett EJ, et al.: Abnormal skeletal muscle capillary recruitment during exercise in patients with type 2 diabetes mellitus and microvascular complications. J Am Coll Cardiol, 2009, 53: 2175-2183. [Medline] [CrossRef]

15) Schrauwen P, Schrauwen-Hinderling V, Hoeks J, et al.: Mitochondrial dysfunction and lipotoxicity. Biochim Biophys Acta, 2010, 1801: 266-271. [Medline] [CrossRef]

16) Sone H, Ito H, Ohashi Y, et al. Japan Diabetes Complication Study Group: Obesity and type 2 diabetes in Japanese patients. Lancet, 2003, 361: 85. [Medline] [CrossRef]

17) Matsuba I, Saito K, Takai M, et al. Japan Diabetes Clinical Data Management Study Group: Fasting insulin levels and metabolic risk factors in type 2 diabetic patients at the first visit in Japan: a 10-year, nationwide, observational study (JDDM 28). Diabetes Care, 2012, 35: 1853-1857. [Medline] [CrossRef]

18) The Japan Diabetes Society: Diabetes Complicated by Obesity, Evidence-based Practice Guideline for the Treatment for Diabetes in Japan 2013. http://www. jds.or.jp/modules/en/index.php?content_id=44 (Accessed Nov. 8, 2016)

19) Kushiyama A, Yoshida Y, Kikuchi T, et al.: Twenty-year trend of increasing obesity in young patients with poorly controlled type 2 diabetes at first diagnosis in urban Japan. J Diabetes Investig, 2013, 4: 540-545. [Medline] [CrossRef]

20) Marcus BH, Simkin LR: The transtheoretical model: applications to exercise behavior. Med Sci Sports Exerc, 1994, 26: 1400-1404. [Medline] [CrossRef]

21) Choi KM: Sarcopenia and sarcopenic obesity. Endocrinol Metab (Seoul), 2013, 28: 86-89. [Medline] [CrossRef]

22) Vermeulen J, Neyens JC, van Rossum E, et al.: Predicting ADL disability in community-dwelling elderly people using physical frailty indicators: a systematic review. BMC Geriatr, 2011, 11: 33. [Medline] [CrossRef]

23) Hasegawa R, Islam MM, Lee SC, et al.: Threshold of lower body muscular strength necessary to perform ADL independently in community-dwelling older adults. Clin Rehabil, 2008, 22: 902-910. [Medline] [CrossRef]

24) Park SW, Goodpaster BH, Strotmeyer ES, et al.: Decreased muscle strength and quality in older adults with type 2 diabetes: the health, aging, and body composition study. Diabetes, 2006, 55: 1813-1818. [Medline] [CrossRef]

25) Park SW, Goodpaster BH, Strotmeyer ES, et al. Health, Aging, and Body Composition Study: Accelerated loss of skeletal muscle strength in older adults with type 2 diabetes: the health, aging, and body composition study. Diabetes Care, 2007, 30: 1507-1512. [Medline] [CrossRef]

26) Ministry of Health Labour and Welfare: Annual Health, Labour and Welfare Report 2013-2014. http://www.mhlw.go.jp/english/wp/wp-hw8/ (Accessed Nov. 8, 2016)

27) Ijzerman TH, Schaper NC, Melai T, et al.: Motor nerve decline does not underlie muscle weakness in type 2 diabetic neuropathy. Muscle Nerve, 2011, 44: 241-245. [Medline] [CrossRef]

28) Nomura T, Ikeda Y, Nakao S, et al.: Muscle strength is a marker of insulin resistance in patients with type 2 diabetes: a pilot study. Endocr J, 2007, 54: 791-796. [Medline] [CrossRef]

29) Yanai H: Nutrition for Sarcopenia. J Clin Med Res, 2015, 7: 926-931. [Medline] [CrossRef] 